\title{
Alternating Copolymerization of Diethyl $\alpha$-Phenylvinyl Phosphate and Maleic Anhydride
}

\author{
Jung-Il JIN and Hong-Ku SHIM \\ Department of Chemistry, College of Sciences, \\ Korea University, 1-Anam Dong, Seoul 132, Korea
}

(Received June 30, 1983)

\begin{abstract}
The kinetics of the free-radical copolymerization of diethyl $\alpha$-phenylvinyl phosphate (DEPVP, $\mathbf{M}_{2}$ ) and maleic anhydride (MAnh, $\mathbf{M}_{1}$ ) in chloroform were studied using benzoyl peroxide as an initiator. The generalized model was successfully applied to determine the reactivity ratios $k_{1 \mathrm{c}} / k_{12}$ and $k_{2 \mathrm{c}} / k_{21}$ from the change in copolymerization rate with monomer feed at a constant total monomer concentration. The copolymerization was found to proceed via two pathways, one involving formation of a charge-transfer complex between the two monomers and the other involving the two free monomers. A kinetic analysis indicated that the rate of formation of the MAnh free radical, $k_{1 \mathrm{c}} k_{21}$, was slightly slower than that of the DEPVP free radical, $k_{2 \mathrm{c}} k_{12}$, and that the copolymerization proceeded predominantly through participation of the free monomers rather than the charge-transfer complex. General kinetic studies at various initiator concentrations showed the overall copolymerization rate to depend on an order of 0.49 with respect to the initiator concentration. The overall activation energy for this copolymerization was found to be $33.5 \mathrm{~kJ} \mathrm{~mol}^{-1}$.
\end{abstract}

KEY WORDS Diethyl $\alpha$-Phenylvinyl Phosphate / Maleic Anhydride / Alternating Copolymerization / Free Radical / Charge-Transfer Complex /

Kinetic Analysis /

The alternating free-radical copolymerization of electron-rich vinyl monomers with MAnh has been the subject of a number of studies, ${ }^{1-7}$ since it is of both synthetic and mechanistic interest. Two different mechanisms have been proposed to explain the formation of $1: 1$ alternating copolymers. One is based on the cross-propagation of free monomers whose transition state is considered to be more stabilized than that of the homo-propagation due to either the difference in polarity between the monomer pair or charge-transfer interactions between a growing polymer radical and a comonomer. ${ }^{8,9}$ The other is the homopolymerization of a chargetransfer complex formed between the monomer pair which usually can be detected spectrophotometrically. ${ }^{10}$

We have been conducting copolymerization studies on a series of vinyl monomers with phosphorus-containing functional groups ${ }^{11-13}$ and described in our earlier paper that diethyl $\alpha$-phenylvinyl phosphate (DEPVP) could form $1: 1$ alternat- ing copolymers with maleic anhydride (MAnh) ${ }^{13}$ Moreover, this monomer pair produced a chargetransfer complex, as was confirmed by NMR spectroscopy. ${ }^{13}$ It was also observed that the rate of copolymerization was maximum at a MAnh mole fraction of about 0.7 . The rate profile obtained by plotting the copolymerization rate against the feed composition was unsymmetric. ${ }^{13}$

Recently Shirota et al. ${ }^{14,15}$ derived a generalized kinetic scheme for $1: 1$ alternating copolymerizations encompassing both the classical terminal model and molecular charge-transfer complex mechanism. This model was recently applied by Javni et al..$^{7}$ to the alternating copolymerization of $\alpha$-methylstyrene and maleic anhydride. This paper describes the results of the application of the same method to the DEPVP-MAnh 1:1 alternating copolymerization system and further elucidates the mechanism through quantitative kinetic analysis. 


\section{EXPERIMENTAL}

\section{Chemicals and Instruments}

The monomer DEPVP was synthesized by refluxing $\alpha$-chloroacetophenone with triethyl phosphite following a literature method. ${ }^{16}$ The DEPVP thus prepared was purified by fractional distillation and had a boiling point of $117-121^{\circ} \mathrm{C}$ at 0.2 torr in agreement with the literature value. ${ }^{16}$ Gas chromatographic analysis of the distilled monomer showed the monomer to be $99.7 \%$ pure. MAnh was purified by vacuum distillation. Benzoyl peroxide (Fisher Scientific), used as the initiator throughout the present work, was dissolved in chloroform and then reprecipitated with ethanol. It was dried at room temperature under vacuum. All other chemicals employed in the present investigation were of reagent or spectroscopic grade and used as received.

Pye Unicam GCD Chromatograph and Unicam SP 500 UV and VIS Spectrophotometer were used in the present investigation.

\section{Copolymerization of Diethyl $\alpha$-Phenylvinyl Phos-} phate (DEPVP) and Maleic Anhydride (MAnh)

All copolymerizations were carried out in solutions using benzoyl peroxide as the initiator. The monomer feed ratio was varied widely at a fixed total monomer concentration. The total monomer concentrations employed were 1.0, 1.5, 2.0, 2.5, and $3.0 \mathrm{moll}^{-1}$, and the initiator concentration was $8.26 \times 10^{-3} \mathrm{moll}^{-1}$. A polymerization solution was placed in a polymerization tube and frozen in a dry ice-acetone bath. A tube was then connected to a vacuum line and the air in the tube was removed by the usual freeze-thaw method. The polymerization tube was then filled with predried nitrogen and placed in a water bath at $70.0 \pm 0.1{ }^{\circ} \mathrm{C}$. Polymerization was stopped by placing the reaction mixture in a dry ice-acetone bath and then mixing with a small quantity of a chloroform solution containing hydroquinone. The polymers formed were precipitated when the chloroform solution was poured into a large volume of diethyl ether. The precipitated polymers were separated by centrifugation and purified by dissolution-precipitation cycles using chloroform and diethyl ether. The polymers obtained were dried under vacuum at $35^{\circ} \mathrm{C}$ to a constant weight.

\section{Determination of Copolymer Composition}

Copolymer compositions were calculated on the basis of their phosphorus content, ${ }^{17,18}$ indicative of DEPVP units, determined calorimetrically. A small quantity $(10-20 \mathrm{mg})$ of copolymer was first oxidized in a hot $1: 1$ mixture of concentrated nitric and sulfuric acids. Further oxidation with perchloric acid and then with hydrogen peroxide resulted in a colorless solution. Reaction with ammonium molybdate and hydrazine sulfate produced a colored solution. Its absorbance was measured at $830 \mathrm{~nm}$ using a UV-VIS spectrophotometer. Tri-para-cresyl phosphate was used as a standard for the construction of a calibration curve. Blank tests were run side by side with actual experiment.

\section{RESULTS AND DISCUSSION}

As reported previously, the DEPVP-MAnh system yielded $1: 1$ alternating copolymers regardless of the monomer feed ratio when using a free-radical initiator. ${ }^{13}$ The equilibrium constant of the chargetransfer (CT) complex between DEPVP and MAnh, determined at $20^{\circ} \mathrm{C}$ by the transformed BenesiHildebrand NMR method ${ }^{3,19}$ in deuterated chloroform, was $0.0851 \mathrm{~mol}^{-1} .^{13}$

The dependence of conversion rates of the equimolar mixtures of the monomer pair on temperature is shown in Figure 1.

The linearity in conversion $v s$. time indicates that the comonomers always react in pairs. This causes the second-order reaction to become pseudo-first-

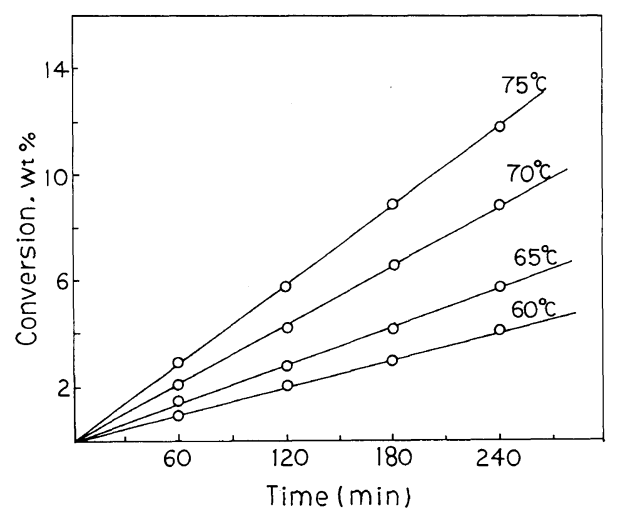

Figure 1. Dependence of conversion on temperature for the copolymerization of DEPVP and MAnh in chloroform. The initial molar ratio of DEPVP to MAnh was $1: 1$. 


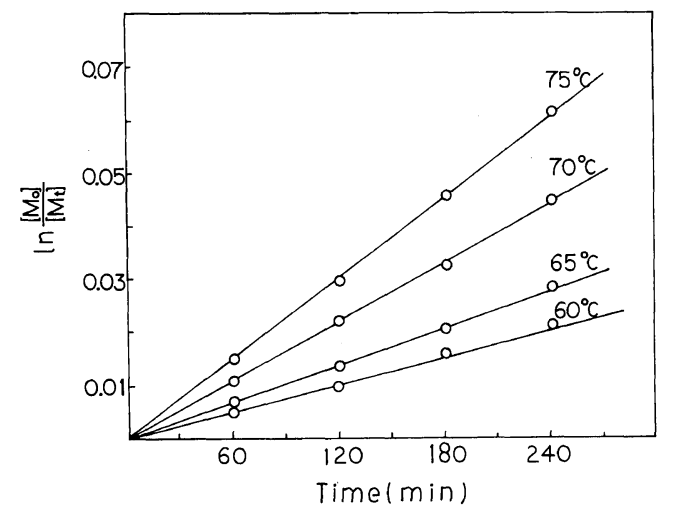

Figure 2. First-order plots for the copolymerization of DEPVP and MAnh in chloroform. The initial molar ratio of DEPVP to MAnh was $1: 1$.

Table I. Rate constants of the copolymerization of DEPVP and MAnh at different temperatures

\begin{tabular}{ccccc}
\hline \multirow{2}{*}{ Exptl. No. } & $\frac{1}{\text { Temp }}$ & & $k$ & $\log k$ \\
\cline { 2 - 3 } \cline { 4 - 5 } & ${ }^{\circ} \mathrm{C}$ & & $\min ^{-1}$ & $\min ^{-1}$ \\
\hline 1 & 60 & $0.9 \times 10^{-4}$ & -4.05 \\
2 & 65 & $1.21 \times 10^{-4}$ & -3.92 \\
3 & 70 & & $1.88 \times 10^{-4}$ & -3.73 \\
4 & 75 & $2.62 \times 10^{-4}$ & -3.58 \\
\hline
\end{tabular}

order, as can be verified by the linear plot of $\ln \left(\left[\mathrm{M}_{0}\right] /\left[\mathrm{M}_{\mathrm{t}}\right]\right)$ against time as shown in Figure 2, where $\left[\mathrm{M}_{0}\right]$ stands for the initial concentration. ${ }^{7}$ Applying the Arrhenius equation ${ }^{7}$ to the data in Table I, an activation energy of $33.5 \mathrm{~kJ} \mathrm{~mol}^{-1}$ was determined for the copolymerization. ${ }^{13}$ The value of the activation energy of this copolymerization system is close to that of $p$-isopropyl- $\alpha$-methylstyrene with maleic anhydride ${ }^{20}$ and lower than those of vinyl ethers with maleic anhydride. ${ }^{7}$

The mechanism of $1: 1$ alternating copolymerization is controversial and several models have been proposed. ${ }^{21,22}$ However, the simultaneous crosspropagation mechanism of uncomplexed free monomers and charge-transfer complex monomers seems to be most widely accepted. The relative reactivities of electron donor and acceptor monomers and the relative degree of participation of free and CT complex monomers in the copolymerization reaction can be quantitatively analyzed from the initial rate of propagation as described by

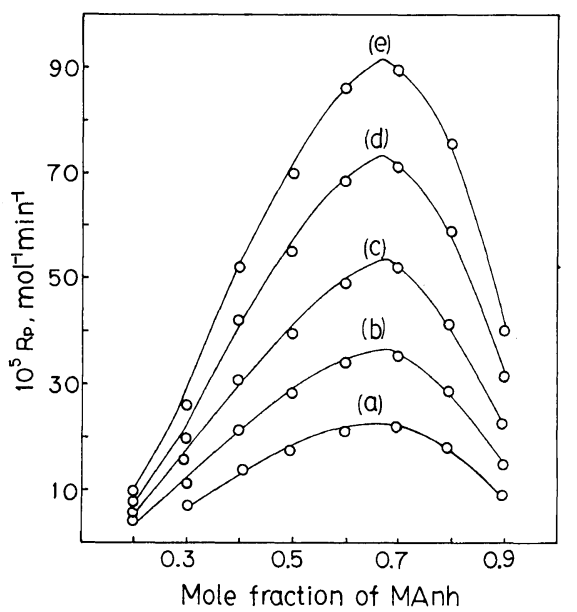

Figure 3. The initial copolymerization rate vs. monomer feed composition in copolymerization of DEPVP and MAnh in chloroform. Total monomer concentration: (a) 1.0 , (b) 1.5 , (c) 2.0 , (d) 2.5 , and (e) 3.0 moll ${ }^{-1}$.

Shirota and co-workers. ${ }^{14,15}$ Their model enables one to evaluate the reactivity ratios from the dependence of the copolymerization rate on the monomer feed composition at a constant total monomer concentration. Figure 3 shows that the maxima of the initial copolymerization rates are always located at higher maleic anhydride concentrations in the feed, at a molar ratio of MAnh : DEPVP of about $7: 3$. Such an unsymmetric rate profile suggests that this copolymerization proceeds through a mixed reaction mechanism involving both a CT complex between the two monomers as well as the free monomers. ${ }^{20,23}$

Assuming that the overall rate of polymerization is given by the sum of copolymerization rate of free monomers $R_{\mathrm{p}}(\mathrm{f})$ and CT-complex monomers $R_{\mathrm{p}}(\mathrm{CT})$ [eq 1] and applying steady-state approximation to the copolymerization, eq 2 can be derived $^{14,15,24}$

$$
\begin{gathered}
R_{\mathrm{p}}=R_{\mathrm{p}}(\mathrm{f})+R_{\mathrm{p}}(\mathrm{CT}) \\
R_{\mathrm{p}} /\left[\mathrm{M}_{1}\right]=A(X) K\left(\frac{k_{1 \mathrm{c}}}{k_{12}}+\frac{k_{2 \mathrm{c}}}{k_{21}} X\right)\left[\mathrm{M}_{1}\right]+A(X)
\end{gathered}
$$

where $K$ is the equilibrium constant of the CTcomplex formation and the subscript $\mathrm{c}$ stands for CT complex between the two monomers, $X=\left[\mathrm{M}_{2}\right] /$ [ $\left.\mathrm{M}_{1}\right]$, and $A(X)$ is given by the following equation: 


$$
A(X)=\frac{2 k_{21} k_{12} R_{\mathrm{i}}{ }^{1 / 2} X}{\left(k_{\mathrm{t} 11} k_{21}{ }^{2}+2 k_{\mathrm{t} 12} k_{21} k_{12} X+k_{\mathrm{t} 22} k_{12}{ }^{2} X^{2}\right)^{1 / 2}}
$$

From the plot of $R_{\mathrm{p}} /\left[\mathrm{M}_{1}\right]$ vs. $\left[\mathrm{M}_{1}\right], A(X) K\left(k_{1 \mathrm{c}} /\right.$ $\left.k_{12}+\left(k_{2 \mathrm{c}} / k_{21}\right) X\right)$ and $A(X)$ can be obtained from the slope of the lines and their intercepts (Figure 4). If the slope is expressed as eq 4 ,

$$
K\left(\frac{k_{1 \mathrm{c}}}{k_{12}}+\frac{k_{2 \mathrm{c}}}{k_{21}} X\right)=F(X)
$$

the plot of $F(X)$ vs. $X$ will give a straight line (Figure 5). The slope of the line corresponds to

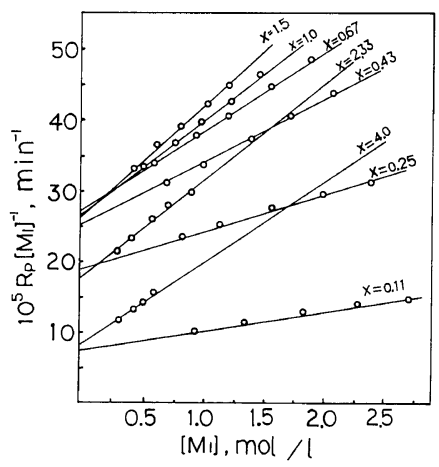

Figure 4. Plot of $R_{\mathrm{p}} /\left[\mathrm{M}_{1}\right]$ vs. $\left[\mathrm{M}_{1}\right]$ at different $\left[\mathrm{M}_{2}\right] /$ $\left[\mathrm{M}_{1}\right]=X$ ratios in the copolymerization of DEPVP and MAnh.

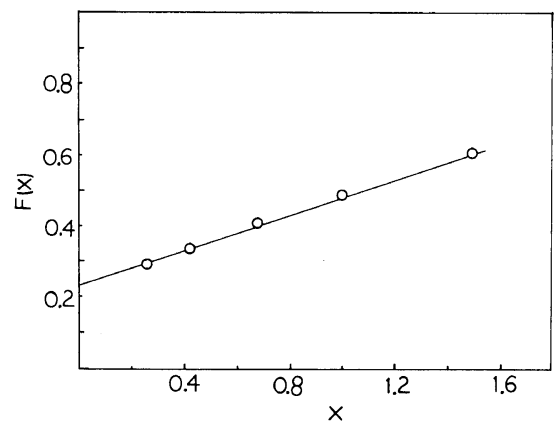

Figure 5. Plot of $F(X)$ vs. $X$ in the copolymerization of DEPVP and MAnh.

Table II. Reactivity ratios of the CT-complex monomers to the free monomers

\begin{tabular}{ccc}
\hline$K\left(k_{1 \mathrm{c}} / k_{12}\right)$ & $K\left(k_{2 \mathrm{c}} / k_{21}\right)$ & $k_{1 \mathrm{c}} k_{21} / k_{2 \mathrm{c}} k_{12}$ \\
\hline 0.231 & 0.253 & 0.913 \\
\hline
\end{tabular}

$K\left(k_{2 \mathrm{c}} / k_{21}\right)$ and the intercept to $K\left(k_{1 \mathrm{c}} / k_{12}\right)$. These values are shown in Table II. The values shown in Table II indicate that the rate of formation of the electron donor free radical, $k_{2 \mathrm{c}} k_{12}$, is almost the same as that of the electron-acceptor free radical, $k_{1 \mathrm{c}} k_{21}$. Since the value of $K$ is much less than 1, these data suggest that the reaction of both monomers with the CT-complex monomer is much faster than the interaction between free monomers.

The relative degree of participation of $R_{\mathrm{p}}(\mathrm{f})$ and $R_{\mathrm{p}}(\mathrm{CT})$ can be calculated using eq 2 and the results
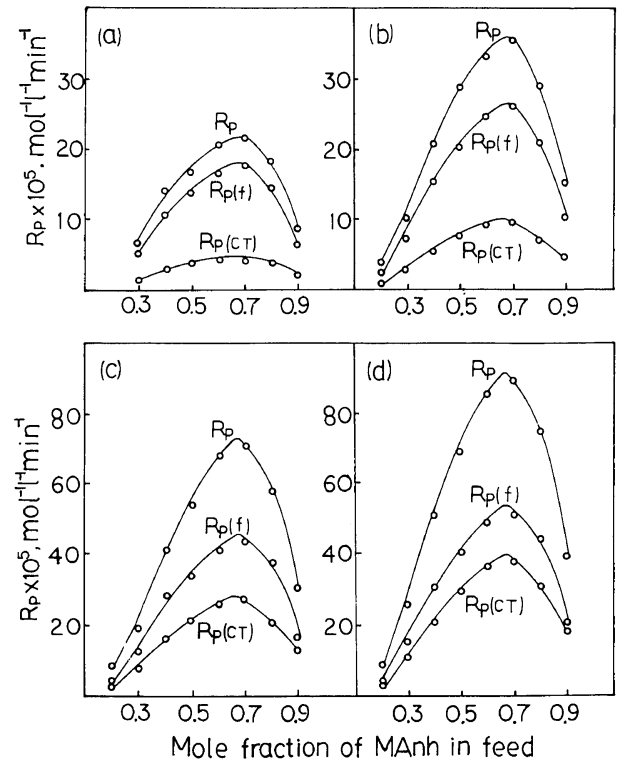

Figure 6. Relative participation of free monomers and CT-complex monomers in the copolymerization of DEPVP and MAnh at different monomer feed compositions. Total monomer concentration: (a) 1.0 (b) 1.5 (c) 2.5 (d) $3.0 \mathrm{moll}^{-1}$.

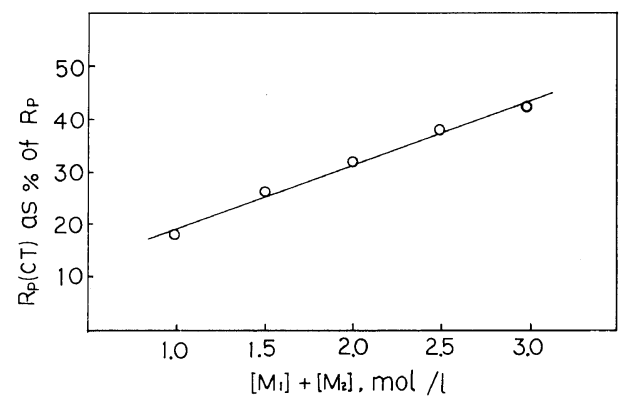

Figure 7. Plot of $R_{\mathrm{p}}(\mathrm{CT})$ against molar concentration of monomers in the copolymerization of DEPVP and MAnh. 


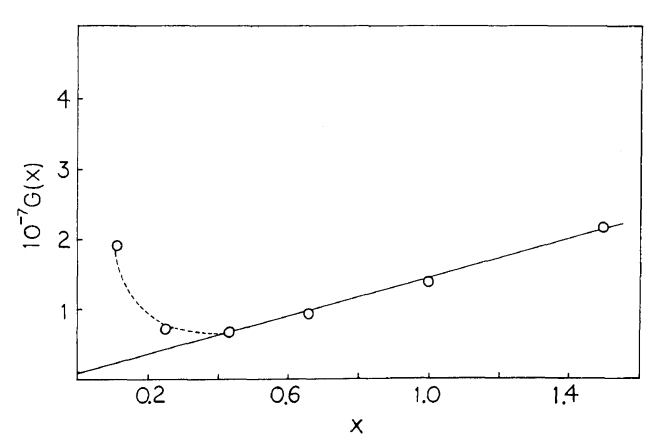

Figure 8. Plot of $G(X)$ vs. $X$ for the copolymerization of DEPVP and MAnh.

Table III. Relative values of termination rate constants for the copolymerization of DEPVP and MAnh

\begin{tabular}{cc}
\hline$\left(1 / 4 R_{\mathrm{i}}\right)\left(k_{\mathrm{t} 22} / k_{21}{ }^{2}\right)$ & $\left(1 / 4 R_{\mathrm{i}}\right)\left(k_{\mathrm{t} 12} / k_{12} k_{21}\right)$ \\
\hline $1.34 \times 10^{7}$ & $0.10 \times 10^{7}$ \\
\hline
\end{tabular}

are graphically presented in Figure 6 . The graphs indicate that the copolymerization proceeds predominantly through the free monomers. The participation of CT-complex monomers, however, increases with an increase in total monomer concentration. This is shown graphically in Figure 7. Information on the termination process can be obtained from the values of $A(X)$ [eq 3] by applying eq 5 :

$$
G(X)=\frac{X}{[A(X)]^{2}}=\frac{1}{4 R_{\mathrm{i}}}\left(\frac{k_{\mathrm{t} 22}}{k_{21}{ }^{2}} X+\frac{2 k_{\mathrm{t} 12}}{k_{21} k_{12}}+\frac{k_{\mathrm{t} 11}}{k_{12}{ }^{2}} \frac{1}{X}\right)
$$

The fact that the plot of $G(X) v s . X$ is linear (Figure 8) shows that $k_{\mathrm{t} 11} / k_{12}{ }^{2}$ is virtually zero. This means the homotermination between polymer radicals with acceptor terminals is negligible. The slope of the line corresponds to $\left(1 / 4 R_{\mathrm{i}}\right)\left(k_{\mathrm{t} 22} / k_{21}^{2}\right)$ and the intercept to $\left(1 / 4 R_{\mathrm{i}}\right)\left(2 k_{\mathrm{t} 12} / k_{21} k_{12}\right)$. These values appear in Table III.

In Figure 8, the two experimental points at low values of $X$ deviate from linearity. This can be ascribed to the fact that the contribution to $G(X)$ of the term, $\left(k_{111} / k_{12}^{2}\right)(1 / X)$, becomes significant as $X$ approaches zero.

The occurrence of bimolecular termination in this system was confirmed by the dependence of the initial copolymerization rate on the initiator concentration to the 0.49 power when the copolymerization was conducted with an equimolar mixture of the monomer pair.

\section{REFERENCES}

1. M. G. Baldwin, J. Polym. Sci., A, 3, 703 (1965).

2. M. L. Hellensleben, Makromol. Chem., 144, 267 (1970).

3. E. J. Goethals, A. Cardon, and R. Grosjean, J. Macromol. Sci., Chem., A7(6), 1265 (1973).

4. R. B. Seymour and D. P. Garner, Polymer, 17, 21 (1976).

5. K. Dodgson and J. R. Ebson, Eur. Polym. J., 13, 791 (1977).

6. R. Vuković, V. Kurešvić, and D. Fleś, J. Polym. Sci., Polym. Chem. Ed., 17, 3935 (1979).

7. I. Javni, D. Fleś, and R. Vuković, J. Polym. Sci., Polym. Chem. Ed., 20, 977 (1982).

8. C. C. Price, J. Polym. Sci., 3, 772 (1948).

9. C. Walling, D. Seymour, and K. B. Wolfstirn, J. Am. Chem. Soc., 70, 1544 (1948).

10. T. Kokubo, S. Iwatsuki, and Y. Yamashita, Macromolecules, 1, 482 (1968); 3, 518 (1970).

11. J.-I. Jin, H.-S. Byun, and S.-M. Lee, J. Macromol. Sci., Chem., A16(5), 953 (1981).

12. J.-I. Jin, H.-K. Shim, and S.-M. Lee, J. Korean Chem. Soc., 26, 6 (1982).

13. J.-I. Jin and H.-K. Shim, J. Korean Chem. Soc., 27, 4 (1983).

14. M. Yoshimura, T. Nogami, M. Yokoyama, H. Mikawa, and Y. Shirota, Macromolecules, 9, 211 (1976).

15. M. Yoshimura, H. Mikawa, and Y. Shirota, Macromolecules, 11, 1085 (1978).

16. I. J. Borowitz, M. Anschel, and S. Firstenberg, J. Org. Chem., 32, 1723 (1967).

17. M. Sander and E. Steininger, Rev. Macromol. Chem., 2, 1 (1967).

18. I. M. Kolthoff et al., "Quantitative Chemical Analysis," 4th ed, Macmillan, London, 1971, p 1126.

19. R. Foster and C. A. Fyee, Trans. Faraday Soc., 61, 1626 (1965).

20. J.-I. Jin, K.-Y. Kim, and H.-S. Byun, Polymer (Korea), 3, 171 (1979).

21. C. Walling, J. Am. Chem. Soc., 71, 1930 (1949).

22. E. Tsuchida and T. Tomono, Makromol. Chem., 141, 265 (1971).

23. Y. Shirota, A. Matsumoto, and H. Mikawa, Polym. J., 3, 643 (1972).

24. Y. Shirota, M. Yoshimura, A. Matsumoto, and H. Mikawa, Macromolecules, 7, 4 (1974). 